\section{Research Square}

Preprints are preliminary reports that have not undergone peer review.

They should not be considered conclusive, used to inform clinical practice, or referenced by the media as validated information.

\title{
Genomic characterization of carbapenem resistant Escherichia coli from multiple hospitals in Nanjing, China: focusing on frequent co-occurrence of blaNDM and blaKPC-2
}

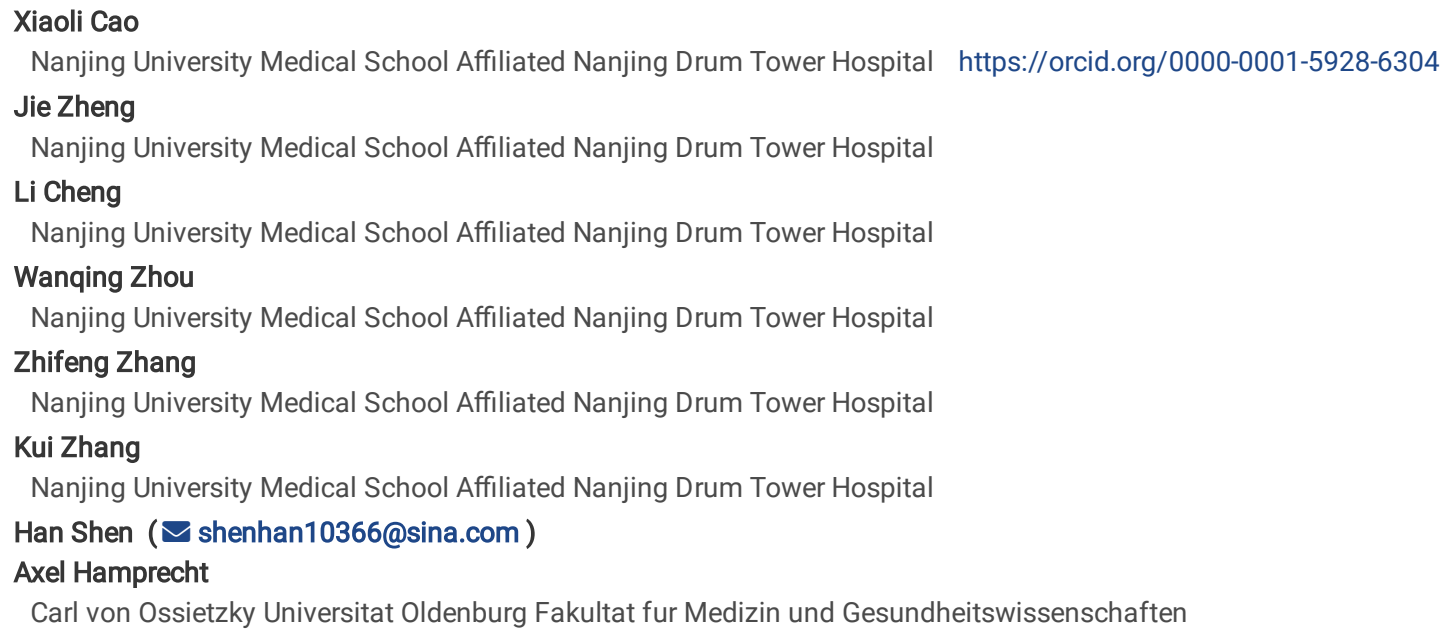




\section{Abstract}

Background: The increasing emergence of carbapenem resistant Escherichia coli (CREC) poses a potential threat to public health, hence genomic characterization of isolates is needed for a better understanding of its transmission and implementation of infection control measures.

Materials and methods区Eleven CREC isolates were collected in 2015 from 6 hospitals in Nanjing, China, and analyzed using whole genome sequencing. Resistance determinants, virulence elements, multi-locus sequence type (MLST), serotypes, phylogeny and fimH types were determined.

Results: All of the CREC carried at least one carbapenemase. NDM-5 ( $n=9)$ was the most frequent carbapenemase, followed by KPC-2 ( $n=3)$ and NDM- $(n=2)$; three isolates produced NDM- 5 and KPC-2. Ten out of the 11 isolates co-carried blaCTX-M variants. MLST analysis found 7 distinct STs, including ST410 $(n=2)$, ST3489 $(n=1)$, ST156 $(n=1)$, ST683 $(n=1)$, ST297 $(n=1)$, ST167 $(n=1)$, and ST361 $(n=1)$. Six distinct serotypes and 8 Fim types were identified. A great diversity of plasmid profiles was observed with plasmid replicon IncX3 being the most frequent $(n=11)$. Phylogenetic analysis showed great diversity between the 11 CREC isolates and also between 6 additional isolates co-carrying blaNDM and blaKPC which were selected from the strains collection of Nanjing Drum Tower Hospital for comparison. Conjugation assays demonstrated that blaNDM was transferable.

Conclusion: NDM is the major carbapenemase among CREC, with NDM-5 being the main variant which can be horizontally disseminated by IncX3 plasmids. These isolates displayed genetic diversity by MLST, Fim typing and serotyping. We herein provided the first report on emergence of NDM-5 producing $E$. coli ST297, ST683, ST3489, and NDM-1 producing E. coliST361.

\section{Introduction}

Escherichia coli mainly inhabits the lower intestinal tract of warm-blooded animals. It is a major pathogen for numerous types of infections, such as intestinal, urinary, and respiratory tract infections in humans and other animals (1). In recent years, carbapenems have been increasingly used as the most effective antibiotic in clinical therapy for infections caused by multidrug-resistant (MDR) strains, due to production of extended spectrum $\beta$-lactamases (ESBLs) or AmpC-type $\beta$-lactamases(2). Therefore, the frequent occurrence of carbapenem-resistant Escherichia coli (CREC) worldwide has been posing a threat to public health $(3,4)$,

Production of carbapenemases has been so far the main mechanism for carbapenem resistance in Enterobacterales(5), and New Delhi metallo- $\beta$-lactamase (NDM) is the major carbapenemase in E. coli all over the world (6). It is worthy to note that the co-occurrence of multiple $ß$-lactamases among single bacteria species (7), especially carbapenemase, such as co-production of NDM-1 and Klebsiella pneumoniae carbapenemase 2 (KPC-2)(8), co-occurrence of KPC-2 and OXA-48, have so far been frequently described in multiple clinical Enterobacterales, such as K. pneumoniae, Enterobacter cloacae, and Citrobacter freundi(8-

10). Thus, such a frequent co-occurrence of carbapenemase in one isolate are causing rapidly rising carbapenem resistance, leading to increasing CREassociated morbidity and mortality (11)

Report from the China CRE Network showed that the overall CREC infection incidence differed significantly by region, with the highest in Shandong (9.1\%) and the lowest in Qinghai (0 \%) during January to December 2015 (12); another study showed a 4.6\% prevalent rate of CREC in in Northern Jiangsu Province, China during September 2015 to August 2016 (13). However, strains analyzed were predominantly collected from tertiary hospitals. Specialized hospitals, Children's hospital and level II hospitals were less involved.

Moreover, multiple studies have shown that that blaNDM is often located on IncX3 plasmids, $(14,15)$, but little information on virulence genes, serotyping and fim typing is available for such strains.

In this study, we tried to characterize the genomic epidemiology of CREC strains including resistance determinants, virulence factors, serotyping, fim typing and plasmid replicons. Furthermore, the molecular characterization of strains co-producing NDM-5 and KPC-2 screened from clinical CREC in our hospital during 2013-2017 were investigated.

\section{Materials And Methods}

\section{Isolates collections}

The isolates in our study included two parts. Firstly, eleven non-repetitive CREC isolates collected from 6 hospitals during the period June-December 2015 in Nanjing city were analyzed for genomic characterization by whole genome sequencing. These strains were isolated from urine ( $n=4)$, sputum ( $n=3)$, blood $(n=1)$, bile $(n=1)$, secretion of uterus neck $(n=1)$, and the source of one strain was unknown. The following hospitals were involved: Nanjing Mingji Hospital I $(n=1)$, Nanjing Children's Hospital $(n=2)$, Nanjing Lishui Hospital $(n=1)$, Nanjing Drum Tower Hospital $(n=4)$, Nanjing jinyu Hospital ( $n=1)$, Nanjing Maternal and Child Health Hospital $(n=1)$, and Nanjing First Hospital $(n=1)$.

Secondly, considering the high prevalence of $E$. coli isolates co-producing KPC-2 and NDM among the 11 CREC strains from 6 hospitals, we tried to investigate the distribution of these strains among clinical CREC isolates. Therefore, a total of 43 consecutive non-duplicate isolates collected in Nanjing Drum Tower hospital during 2013-2017 were further analyzed for isolates co-producing KPC-2 and NDM by PCR and DNA sequencing. Among them, 4 strains were isolated in 2013, 10 in 2014, 2 in 2015, and 11 from 2016 and 16 in 2017. The source of the samples was as follows: urine ( $n=18)$, blood ( $n=9)$, sputum ( $n=6)$, secretion $(n=3)$, bile $(n=3)$, abdominal dropsy $(n=2)$, and pus $(n=1)$.

All the CREC strains were identified by Vitek 2.0 (BioMérieux. Marcy l'Etoile, France) or ATB 32E Semi-auto identification machine (Bio-Mérieux, France). Isolates resistant to at least one carbapenem (imipenem, meropenem, ertapenem) were included in the study. 
Susceptibility of the 11 CREC isolates towards antimicrobial agents were tested by micro-broth method. The antimicrobial agents tested included ertapenem, imipenem, meropenem, cefepime, ceftazidime, cefotaxime, cefuroxime, cefazolin, piperacillin/tazobactam, amikacin, gentamicin, funantuoyin, trimethoprim and sulphame-thoxazole, aztreonam, piperacillin, ciprofloxacin, levofloxacin, aztreonam/avibactam, ceftazidime/avibactam, tigecycline, and colistin B. E. coli ATCC 25922 was used as quality control. The results were interpreted according to the CLSI 2019 guideline (16). Whereas, for tigecycline and colistin, the European Committee on Antimicrobial Susceptibility Testing breakpoints were referred (http://www.eucast.org/clinical_breakpoints).

\section{DNA Extraction}

The Ultraclean Microbial DNA Isolation Kit (MOBIO Laboratories, Carlsbad, CA, US) was used to extract genomic DNA. The NanoDrop 2000c spectrophotometer (Thermo Scientific, Waltham, MA, USA) was used for measuring the DNA concentration and purity for whole genome sequencing.

\section{Whole Genome Sequencing, denovo Assembly, Scaffolding, and Annotation}

The prepared pair-end DNA library was sequenced on the MiSeq (Illumina, SanDiego, CA, USA). Denovo assembly of the paired-end reads was performed by CLC Genomics Workbenchv7.0.4 (QIAGEN, Hilden, Germany) after quality trimming (Qs $\geq 20$ ). Scaffolding was finished using SSPACE standard version 3.0 and the gaps within scaffolds were further closed by GapFiller $(17,18)$. Then genomes were then submitted to NCBI for annnotation.

\section{Analysis of genomic epidemiology}

The antimicrobial resistance determinants and virulence factors were identified using Resfinder v2.1 (http://cge.cbs.dtu.dk/services/ResFinder-2.1/) and Virulence Finder 2.0 (https://cge.cbs.dtu.dk/services/VirulenceFinder/); the 11 CREC were typed by multi-locus sequence typing (MLST) 2.0 (https://cge.cbs.dtu.dk/services/MLST/), Plasmid Finder 2.1 (https://cge.cbs.dtu.dk/services/PlasmidFinder/), Serotype Finder 2.0 (https://cge.cbs.dtu.dk/services/SerotypeFinder/), and FimTyper 1.0 (https://cge.cbs.dtu.dk/services/FimTyper/).

\section{Phylogenetic relationship of 11 CREC}

The core-genome phylogeny of the 11 CREC was constructed by using single-nucleotide polymorphisms (SNP)-sites detected from 1010 core genes (identity > $95 \%$; coverage $=100 \%$ ) derived from 502 ST11 strains (19). A maximum-likelihood tree was calculated using RAxML version 8.2.8, with general timereversible model and 100 bootstraps (20). Interactive Tree Of Life (https://itol.embl.de) was used to produce the phylogenetic tree (21).

\section{The screening of Escherichia coli co-producing NDM and KPC-2 carbapenemases}

In order to investigate the prevalence of $E$. coli isolates co-producing NDM-5 and KPC-2 in our hospital during 2013-2017, genes encoding carbapenemases (KPC and NDM) were detected by PCR and DNA sequencing (22). The positive products were sent to the Qingke Biotechnology Co., Ltd (Nanjing, China) for purification and sequencing. Sequences were further analyzed by using the Chromas-Pro application and BLAST (www.ncbi.nlm.nih.gov/BLAST).

\section{Pulsed-field gel electrophoresis}

Six E. coli isolates co-producing NDM-5 and KPC-2 including 3 ones from 11 CREC and 3 ones selected from the 43 CREC were further analyzed for genetic relatedness by PFGE, which was performed according to the protocol as previously described (23). Briefly, fresh colonies were mixed with proteinase $\mathrm{K}$ (Merck Sharp \& Dohme Ltd, Germany) into plugs. After the plugs were digested by restriction endonuclease Xbal (Fermentas, ABI, Germany), the resultant DNA fragments were separated in a PFGE CHEF-DR III system (Bio-Rad Laboratories, Hercules, CA) in 0.5xTris-borate-EDTA buffer at $120 \mathrm{~V}$ for 19 h. The pulse times ranged from $2.2 \mathrm{~s}$ to $54.2 \mathrm{~s}$. Finally, the BioNumerics software (Applied Math, Sint-Maten-Latem, Belgium) was used to analyze the banding patterns.

\section{Conjugation assay}

For the 6 isolates co-carrying blaKPC and blaNDM, broth mating was performed in order to analyze the transferability of these genes according to the protocol prescribed previously(24). Azide resistant $E$. coli J53 was used as the recipient. Briefly, fresh colonies were inoculated into $5 \mathrm{ml}$ LB broth and incubated at $37^{\circ} \mathrm{C}, 200 \mathrm{rpm}$. After 5 hours, $500 \mu \mathrm{l}$ recipient cells and $100 \mathrm{ul}$ donor were suspended in $5 \mathrm{ml}$ LB broth for overnight culture at $37^{\circ} \mathrm{C}, 200 \mathrm{rpm}$, then $100 \mu \mathrm{l}$ were plated onto the LB plates containing $30 \mathrm{mg} / \mathrm{L}$ cefoxitin and $100 \mathrm{mg} / \mathrm{L}$ sodium azide for E. coli J53. PCR (amplification for blaNDM and blaKPC) and Eric-PCR were used to verify conjugants.

\section{Results}

\section{The susceptibilities of the 11 CREC.}

All the 11 CREC isolates displayed resistance toward $\beta$-lactams (including carbapenems), $\beta$-lactamase inhibitors (including ceftazidime/avibactam) and fluoroquinolones tested in our study. Resistance to trimethoprim and sulphame-thoxazole (72.7, 8/11), gentamicin (36.4, 4/11), amikacin (18.1, 2/11) as well as funantuoyin $(9.1 \%, 1 / 11)$ were also observed. All the 11 isolates were susceptible to tigecycline, colistin and aztreonam/avibactam (Table 1$)$. Compared to the minimum inhibitory concentrations (MICs) of strains co-carrying blaKPC and blaNDM, the isolates only carrying blaNDM did not show obviously higher MICs toward all the antimicrobial agents.

\section{Genomic epidemiology of 11 CREC}


The genomic features including the sizes of genome, GC content and accession number of the 11 CREC isolates were shown in Table 2. Genomic analysis showed that all the 11 CREC strains carried NDM carbapenemases, including 2 NDM-1 and 9 NDM-5, while three strains co-carried NDM-5 and KPC-2; ten out of the 11 isolates carried CTX-M variants, including CTX-M-55 ( $\mathrm{n}=4)$, CTX-M-65 (n=2), CTX-M-14 ( $\mathrm{n}=2)$ and CTX-M-15 ( $\mathrm{n}=2)$. AmpC enzyme including 2 CMY-2 and 1 CMY-43, plasmid mediated quinolone resistance (PMQRs), including oqXAB $(\mathrm{n}=2)$, qnrA1 $(\mathrm{n}=1)$, qnrS1 $(\mathrm{n}=4)$, aac $\left(6^{\prime}\right) \operatorname{lb}-\mathrm{cr}(\mathrm{n}=4)$, and $q e p A(\mathrm{n}=1)$, as well as plasmid-mediated glutathione S-transferase (PMGST) genes fos $A 3(\mathrm{n}=4)$ were also identified (Figure 1).

Virulence genes analysis showed that 12 VFs were detected, with gad $(\mathrm{n}=9)$ being the most frequent one, followed by IpfA ( $\mathrm{n}=7)$ and iss ( $\mathrm{n}=7)$, other VFs including astA $(\mathrm{n}=3), \operatorname{cma}(\mathrm{n}=3), \operatorname{cap} U(\mathrm{n}=3)$, iro $N(\mathrm{n}=2)$, sat $(\mathrm{n}=1), \operatorname{sen} B(\mathrm{n}=1), \operatorname{sep} A(\mathrm{n}=1)$, iha $(\mathrm{n}=1)$ and $\operatorname{cnf} 1(\mathrm{n}=1)$ were also found $(\operatorname{Table} 3)$.

MLST analysis found 7 distinct STs, including ST410 (N=2), ST3489 ( $n=1)$, ST156 ( $=1)$, ST683 ( $n=1)$, ST297 ( $n=1)$, ST167 ( $n=1)$ and ST361 ( $=1)$, in addition, 3 new STs were identified (Figure 1);

The 11 strains were typed into 5 E. coli $\mathrm{O}$ groups $(08,09,025,030,089)$ and $8 \mathrm{H}$ groups $(\mathrm{H} 9, \mathrm{H} 10, \mathrm{H} 4, \mathrm{H} 21, \mathrm{H} 40, \mathrm{H} 45, \mathrm{H} 30$ and $\mathrm{H} 26)$. Six distinct serotypes were found including 08:H9 (n=3), 09:H4 (n=1), 089:H9 (n=1), 030:H21 (n=1), 025:H26 (n=1) and 09:H30 (n=1).

Analysis of FimH identified 8 types, including Fim24 (n=2), Fim23 (n=2), Fim34 (n=1), Fim38 (n=1), Fim121 (n=1), Fim276 (n=1), Fim54 (n=1) and Fim31 $(n=1)$.

A great diversity of plasmid profiles was observed. Among them, plasmid replicon IncX3 was found among all the 11 strains followed by IncFII ( $\mathrm{n}=7$ ) and $\operatorname{IncFIB}(n=6)$, additionally, Incl1 $(n=3), \operatorname{IncFIA}(n=3), \operatorname{IncY}(n=2)$ and IncFIC $(n=2)$ were also detected.

\section{Phylogenetic characterization of 11 CREC strains}

The phylogenetic tree showed that 11 CREC evolve into 2 main clades albeit a great diversity was observed (Figure 1). Two isolates from Nanjing Children's hospital displayed close evolutionary relationship.

\section{Prevalence of isolates co-producing NDM-5 and KPC-2}

Among the 43 CREC collected from our hospital during 2013-2017, 6 KPC-2 and 23 NDM were identified, two strains co-producing NDM-5 and KPC-2 and one strain co-carrying NDM-1 and KPC-2 were found. One was isolated from the urine of an inpatient in ICU in 2014, the other two strains were isolated from abdominal dropsy and sputum of the different patients in 2017.

\section{Genetic relatedness of strains co-carrying blaKPC and blaNDM}

PFGE displayed a high diversity of the 6 strains co-producing blaKPC and blaNDM (Figure 2), indicating that these strains were not from the same clone.

\section{Transferability of blaKPC and blaNDM}

Conjugation assay revealed that the blaNDM of all the 6 isolates was transferable to E. coli J53. However, we could not isolate any conjugants with blaKPC, suggesting that the blaKPC and blaNDM were not on the same plasmid.

\section{Discussion}

In this study, we provided data on genomic epidemiology of 11 CREC strains from 6 hospitals in Nanjing city, Jiangsu province. Based on the high cooccurrence of blaNDM-5 and blaKPC-2, 43 CREC strains collected from a tertiary hospital during 2013-2017 were further screened to investigate the prevalence of such strains in our hospital. This is the first study that provided the genomic epidemiology of the CREC from multiple hospitals in Nanjing.

The high resistance toward the commonly used antibiotics in clinical therapy displayed by the CREC from 6 hospitals were consistent with the previous report (25), leading to a quite limited choice of antimicrobial agents for infections caused by such strains. Fortunately, tigecycline, colistin and aztreonam/avibactam showed the best sensitivity. Of note, the co-occurrence of KPC and NDM among single isolate seems not confer higher resistance to $\beta$-lactams when the MICs of $\beta$-lactams were compared between the strains carrying blaNDM and the ones co-carrying blaKPC and blaNDM. In addition, it was reported that MICs of ertapenem against strains producing NDM-5 are 4- or 8-fold higher than those against strains producing NDM-1(26), however, we did not observe such a phenomenon, we therefore speculate that the existence of other resistant mechanism such as the production of ESBLs and AmpCs, overexpressed efflux pumps, as well as decreased outer membrane permeability may contribute to the resistance towards $\beta$-lactams.

The high prevalence of NDM among 11 CREC in our study is in accordance with the previous report (6), indicating that NDM is the major carbapenemase for carbapenem resistance in $E$. coli, which may result from the low fitness burden of the plasmid harboring b/aNDM in E. coli (27). We found that NDM-5 is the most common variant, this is also in agreement with the present epidemiological data (28), demonstrating that NDM-5 is the predominant determinant conferring carbapenem resistance in CREC. Noteworthily, NDM-5 has been predominantly found in high-risk clone ST167, ST410 and ST101 in the hospital (29).This may result from successful expansion of $E$. coli clonal groups and frequent horizontal gene transfer of NDM-5 expressing plasmids. Notetaceblily, ST131 as a multidrug clone has spread extensively throughout the world (30). However, ST131 was not detected in our study. The multiple distinct STs identified in our study indicated the diversity of these CREC, which was also confirmed by the phylogenetic relationship. As known, NDM-5 has been reported in E. coli ST410, ST156, and ST167 (24). However, to the best of our knowledge, NDM-5 producing E. coli ST297, ST683 and ST3489, as well as NDM-1 producing E. coliST361 has not been reported previously. 
Moreover, we found a high co-occurrence of KPC-2 and NDM-5, 5 out of the 6 strains that were isolated from our hospital which is a comprehensive tertiary hospital with 3000 beds. The more worse is that multiple resistance determinants including blaOXA-1, blaCMY, blaCTX-M, and fosA3, rmtB, qnr and aac(6')-Ibcrwere also identified in these NDM-5 and/or KPC-2 producing strains, representing a significant challenge for clinical management and public health.

Despite multiple plasmid replicons were found among our CREC, most blaNDM -carrying plasmids belong to limited replicon types (IncX3, IncFII, or IncC)(4)

Considering that plasmid replicon InX3 was found among all the NDM-producing E. coli, we speculate that IncX3 is the main host for blaNDM (31). Note worthily, the conjugation assay in our study showed that the spread of blaNDM was not accompanied by transfer of blaKPC-2, indicating that blaNDM and blaKPC-2 were not harbored by the same plasmid.

Virulence gene analysis revealed several major VFs in CREC, among them, gad encodes glutamate decarboxylase, which is the structural component of the major acid resistance system that protects $E$. coli from strong acid stress $(\mathrm{pH}<3)$, typically encountered in the mammalian gastrointestinal tract (32), IpfA (Long polar fimbriae) is a putative adhesion gene, encoding one of the few fimbrial adhesions of enterhemorrhagic E. coli 0157:H7 associated with colonization on host intestine, which play essential roles during the bacterial infection process(32). iss (increased serum survival) is the most common avian pathogenic E. coli encoding gene. It has been identified as a virulence trait associated with the virulence of $E$. coli, causing colibacillosis in poultry (33). The high prevalence of these VFs among CREC may suggest that CREC mainly colonize the host intestine, and they might have a lower potential to cause human disease. Noteworthy, a strain isolated from urine belong to a new ST, which not only carried gad and iss, IpfA, but also sat (secreted autotransporter toxin), $\operatorname{sen} B$ (Plasmid-encoded enterotoxin), sepA (Shigella extracellular protein A), iha (Adherence protein) and cnf1 (Cytotoxic necrotizing factor). It is known that sat can promote cytotoxic effects in several lines of undifferentiated epithelial cells and is highly prevalent in certain $E$. coli pathogenic groups responsible for urinary and intestinal infections(34). Cnf1 is frequently expressed in clinical UPEC isolates, CNF1-producing and $\beta$-hemolytic E. coli strains most notably cause urinary tract and meningeal infections in humans(35). Altogether, the wide presence of these VFs among the urinary CREC may indicate a higher pathogenicity of this strain.

08:H9 as the most common serotype in our study was consistent with previous report, indicating that 08:H9 is a clinically-relevant serotype correlated with multidrug resistance(36)

Altogether, the multiple fimH types, the diverse STs and serotypes in our study, indicates a high diversity of these CREC strains. Although further PFGE from the strains co-carrying KPC and NDM excludes an epidemic dissemination of the frequent occurrence of these strains, the emergence of these strains poses a potential public health threat.

In conclusion, genomic analysis found that NDM is the main carbapenemase for 11 CREC strains, with a frequent occurrence of KPC. These CREC strains displayed genetic diversity by MLST, Fim typing and serotyping, as well as the phylogenetic relationship. IncX3 may be the main plasmid for NDM, and gad, iss and IpfA were the main VFs; Albeit a frequent occurrence of strains co-carrying blaNDM and blaKPC-2 were detected, no clonal dissemination was found; based on the potential public health threat posed by these strains, infection control measures should be further strengthened.

\section{Declarations}

\section{Acknowledgments}

This study was supported by the Natural Science Foundation of Jiangsu Province (Grant No. BK20140099), Nanjing Medical Science and technique Development Foundation (QRX17059) and National Natural Science Foundation of China (81902124).

Conflict of interest statement

None declared

\section{References}

1. Jang J, Hur HG, Sadowsky MJ, Byappanahalli MN, Yan T, Ishii S. Environmental Escherichia coli: ecology and public health implications-a review. 2017;123(3):570-81.

2. Pana ZD, Zaoutis T. Treatment of extended-spectrum beta-lactamase-producing Enterobacteriaceae (ESBLs) infections: what have we learned until now? 2018;7.

3. Mitgang EA, Hartley DM, Malchione MD, Koch M, Goodman JL. Review and mapping of carbapenem-resistant Enterobacteriaceae in Africa: Using diverse data to inform surveillance gaps. International journal of antimicrobial agents. 2018;52(3):372-84.

4. Wu W, Feng Y. NDM Metallo-beta-Lactamases and Their Bacterial Producers in Health Care Settings. 2019;32(2).

5. Patel G, Bonomo RA. "Stormy waters ahead": global emergence of carbapenemases. Front Microbiol. 2013;4:48.

6. Nordmann P, Poirel L, Walsh TR, Livermore DM. The emerging NDM carbapenemases. Trends Microbiol. 2011;19(12):588-95.

7. Guducuoglu H, Gursoy NC, Yakupogullari Y, Parlak M, Karasin G, Sunnetcioglu M, et al. Hospital Outbreak of a Colistin-Resistant, NDM-1- and OXA-48Producing Klebsiella pneumoniae: High Mortality from Pandrug Resistance. Microb Drug Resist. 2018;24(7):966-72.

8. Yu J, Tan K, Rong Z, Wang Y, Chen Z, Zhu X, et al. Nosocomial outbreak of KPC-2- and NDM-1-producing Klebsiella pneumoniae in a neonatal ward: a retrospective study. BMC infectious diseases. 2016;16(1):563.

9. Ben Tanfous F, Alonso CA, Achour W, Ruiz-Ripa L, Torres C, Ben Hassen A. First Description of KPC-2-Producing Escherichia coli and ST15 OXA-48-Positive Klebsiella pneumoniae in Tunisia. Microb Drug Resist. 2017;23(3):365-75.

Page $5 / 9$ 
10. Tada T, Tsuchiya M, Shimada K, Nga TTT, Thu LTA, Phu TT, et al. Dissemination of Carbapenem-resistant Klebsiella pneumoniae clinical isolates with various combinations of Carbapenemases (KPC-2, NDM-1, NDM-4, and OXA-48) and 16S rRNA Methylases (RmtB and RmtC) in Vietnam. BMC Infect Dis. 2017;17(1):467.

11. Potter RF, D'Souza AW, Dantas G. The rapid spread of carbapenem-resistant Enterobacteriaceae. Drug Resist Updat. 2016;29:30-46.

12. Zhang Y, Wang Q, Yin Y, Chen H, Jin L, Gu B, et al. Epidemiology of Carbapenem-Resistant Enterobacteriaceae Infections: Report from the China CRE Network. Antimicrob Agents Chemother. 2018;62(2).

13. Bi R, Kong Z, Qian H, Jiang F, Kang H, Gu B, et al. High Prevalence of bla (NDM) Variants Among Carbapenem-Resistant Escherichia coli in Northern Jiangsu Province, China. Front Microbiol. 2018;9:2704.

14. Mouftah SF, Pal T, Darwish D, Ghazawi A, Villa L, Carattoli A, et al. Epidemic IncX3 plasmids spreading carbapenemase genes in the United Arab Emirates and worldwide. Infection and drug resistance. 2019;12:1729-42.

15. Khalid S, Ahmad N, Ali SM, Khan AU. Outbreak of Efficiently Transferred Carbapenem-Resistant blaNDM-Producing Gram-Negative Bacilli Isolated from Neonatal Intensive Care Unit of an Indian Hospital. Microbial drug resistance (Larchmont, NY). 2019.

16. CLSI. Performance Standards for Antimicrobial Susceptibility Testing. CLSI. 2019;M100-S25.

17. Boetzer M, Henkel CV, Jansen HJ, Butler D, Pirovano W. Scaffolding pre-assembled contigs using SSPACE. Bioinformatics. 2011;27(4):578-9.

18. Boetzer M, Pirovano W. Toward almost closed genomes with GapFiller. Genome biology. 2012;13(6):R56.

19. Page AJ, Taylor B, Delaney AJ, Soares J, Seemann T, Keane JA, et al. SNP-sites: rapid efficient extraction of SNPs from multi-FASTA alignments. Microb Genom. 2016;2(4):e000056.

20. Stamatakis A. Using RAxML to Infer Phylogenies. Current protocols in bioinformatics. 2015;51:6.14.1-.

21. Letunic I, Bork P. Interactive Tree Of Life (iTOL) v4: recent updates and new developments. Nucleic acids research. 2019.

22. Dallenne C, Da Costa A, Decre D, Favier C, Arlet G. Development of a set of multiplex PCR assays for the detection of genes encoding important betalactamases in Enterobacteriaceae. The Journal of antimicrobial chemotherapy. 2010;65(3):490-5.

23. Ribot EM, Fair MA, Gautom R, Cameron DN, Hunter SB, Swaminathan B, et al. Standardization of pulsed-field gel electrophoresis protocols for the subtyping of Escherichia coli 0157:H7, Salmonella, and Shigella for PulseNet. Foodborne pathogens and disease. 2006;3(1):59-67.

24. Bi R, Kong Z, Qian H, Jiang F, Kang H, Gu B, et al. High Prevalence of bla NDM Variants Among Carbapenem-Resistant Escherichia coli in Northern Jiangsu Province, China. Frontiers in microbiology. 2018;9:2704.

25. Li X, Fu Y, Shen M, Huang D, Du X, Hu Q, et al. Dissemination of bla(NDM-5) gene via an IncX3-type plasmid among non-clonal Escherichia coli in China. Antimicrob Resist Infect Control. 2018;7:59.

26. Hornsey M, Phee L, Wareham DW. A novel variant, NDM-5, of the New Delhi metallo- $\beta$-lactamase in a multidrug-resistant Escherichia coli ST648 isolate recovered from a patient in the United Kingdom. Antimicrob Agents Chemother. 2011;55(12):5952-4.

27. Gottig S, Riedel-Christ S, Saleh A, Kempf VA, Hamprecht A. Impact of blaNDM-1 on fitness and pathogenicity of Escherichia coli and Klebsiella pneumoniae. International journal of antimicrobial agents. 2016;47(6):430-5.

28. Wu W, Feng Y, Tang G, Qiao F, McNally A, Zong Z. NDM Metallo- $\beta$-Lactamases and Their Bacterial Producers in Health Care Settings. Clin Microbiol Rev. 2019;32(2).

29. Sun P, Xia W, Liu G, Huang X, Tang C, Liu C, et al. Characterization Of bla (NDM-5)-Positive Escherichia coli Prevalent In A University Hospital In Eastern China. Infect Drug Resist. 2019;12:3029-38.

30. Mathers AJ, Peirano G, Pitout JD. Escherichia coli ST131: The quintessential example of an international multiresistant high-risk clone. Adv Appl Microbiol. 2015;90:109-54.

31. Wang Y, Tong MK, Chow KH, Cheng VC, Tse CW, Wu AK, et al. Occurrence of Highly Conjugative IncX3 Epidemic Plasmid Carrying bla NDM in Enterobacteriaceae Isolates in Geographically Widespread Areas. Frontiers in microbiology. 2018;9:2272.

32. Giovannercole F, Mérigoux C, Zamparelli C, Verzili D, Grassini G, Buckle M, et al. On the effect of alkaline pH and cofactor availability in the conformational and oligomeric state of Escherichia coli glutamate decarboxylase. Protein Eng Des Sel. 2017;30(3):235-44.

33. Xu WY, Li YJ, Fan C. Different loci and mRNA copy number of the increased serum survival gene of Escherichia coli. Can J Microbiol. 2018;64(2):147-54.

34. Toloza L, Gimenez R, Fabrega MJ, Alvarez CS, Aguilera L, Canas MA, et al. The secreted autotransporter toxin (Sat) does not act as a virulence factor in the probiotic Escherichia coli strain Nissle 1917. BMC microbiology. 2015;15:250.

35. Landraud L, Gauthier M, Fosse T, Boquet P. Frequency of Escherichia coli strains producing the cytotoxic necrotizing factor (CNF1) in nosocomial urinary tract infections. Lett Appl Microbiol. 2000;30(3):213-6.

36. Amezquita-Lopez BA, Quinones B. Antimicrobial resistance profiles of Shiga toxin-producing Escherichia coli 0157 and Non-0157 recovered from domestic farm animals in rural communities in Northwestern Mexico. 2016;5:1.

\section{Tables}

Table 1. The minimum inhibitory concentrations of the 11 carbapenem resistant Escherichia coli isolates from 6 hospitals in Nanjing city, Jiangsu province 


\begin{tabular}{|c|c|c|c|c|c|c|c|c|c|c|c|c|c|c|c|c|c|c|}
\hline Strains & ETP & IPM & MEM & FEP & CAZ & CTX & CXM & CZL & TZP & AMK & GEN & NIT & SXT & ATM & PIP & CIP & LVX & A \\
\hline NJMJYY4 & 32 & $>16$ & $>16$ & $>32$ & $>32$ & $>32$ & $>64$ & $>32$ & $>256 / 4$ & $<1$ & $<1$ & 64 & $<0.25$ & 32 & 256 & $>8$ & 16 & $<1$ \\
\hline NJSETYYY11 & $>32$ & $>16$ & $>16$ & $>32$ & $>32$ & $>32$ & $>64$ & $>32$ & $>256 / 4$ & $<1$ & $<1$ & 16 & $>32$ & $>128$ & $>256$ & $>8$ & $>16$ & 1 \\
\hline $\begin{array}{l}\text { NJSETYYY } \\
14\end{array}$ & $>32$ & $>16$ & $>16$ & $>32$ & $>32$ & $>32$ & $>64$ & $>32$ & $>256 / 4$ & $<1$ & $<1$ & 32 & $>32$ & $>128$ & $>256$ & $>8$ & 16 & 1 \\
\hline NJSFYYY17 & $>32$ & $>16$ & $>16$ & $>32$ & $>32$ & $>32$ & $>64$ & $>32$ & $>256 / 4$ & 1 & 2 & $>128$ & $>32$ & $>128$ & $>256$ & $>8$ & $>16$ & 1 \\
\hline NJLSYY40 & $>32$ & $>16$ & $>16$ & $>32$ & $>32$ & $>32$ & $>64$ & $>32$ & $>256 / 4$ & $<1$ & 64 & 8 & $>32$ & 64 & $>256$ & $>8$ & $>16$ & $<1$ \\
\hline NJSDYYY42 & 16 & $>16$ & $>16$ & $>32$ & $>32$ & $>32$ & $>64$ & $>32$ & $>256 / 4$ & $>128$ & $>128$ & 16 & $>32$ & 64 & $>256$ & 2 & 4 & 1 \\
\hline NJJYYY51 & $>32$ & $>16$ & $>16$ & $>32$ & $>32$ & $>32$ & $>64$ & $>32$ & $>256 / 4$ & $>128$ & $>128$ & 32 & $>32$ & $>128$ & $>256$ & $>8$ & 16 & 0 \\
\hline NJGLYY3610 & 8 & 16 & $>16$ & $>32$ & $>32$ & $>32$ & $>64$ & $>32$ & $128 / 4$ & 2 & 16 & 8 & 320 & 64 & $>256$ & 4 & 8 & 1 \\
\hline $\begin{array}{l}\text { NJGLYY } \\
3940\end{array}$ & $>32$ & $>16$ & $>16$ & $>32$ & $>32$ & $>32$ & $>64$ & $>32$ & $>256 / 4$ & $<1$ & $<1$ & 16 & $>32$ & 32 & $>256$ & $>8$ & 16 & $<1$ \\
\hline $\begin{array}{l}\text { NJGLYY } \\
4197\end{array}$ & $>32$ & $>16$ & $>16$ & $>32$ & 32 & $>32$ & $>64$ & $>32$ & $256 / 4$ & $<1$ & $<1$ & 32 & $<0.25$ & $>128$ & $>256$ & 2 & $>16$ & 1 \\
\hline $\begin{array}{l}\text { NJGLYY } \\
4673\end{array}$ & $>32$ & $>16$ & $>16$ & $>32$ & $>32$ & $>32$ & $>64$ & $>32$ & $>256 / 4$ & 1 & 2 & 8 & $<0.25$ & $>128$ & $>256$ & $>8$ & $>16$ & 0 \\
\hline
\end{tabular}

ETP, ertapenem; IPM, imipenem; MEM, meropenem; FEP, cefepime; CAZ, ceftazidime; CTX, cefotaxime; CXM, cefuroxime; CZL, cefazolin; TZP, piperacillin/tazobactam; AMK, amikacin; GEN, gentamicin; NIT, funantuoyin; SXT, trimethoprim and sulphame-thoxazole; ATM, aztreonam; PIP, piperacillin; CIP, ciprofloxacin; LVX, levofloxacin; AZA, aztreonam/avibactam; CZA, ceftazidime/avibactam; TGC, tigecycline; COL, colistin B.

Table 2. The genome features of the 11 carbapenem resistant Escherichia coli isolates from 6 hospitals in Nanjing city, Jiangsu province.

\begin{tabular}{|lllll|}
\hline Sample & Number of contigs & Total length (bp) & GC\% & Accession number \\
\hline NJJYYY51 & 148 & 5308317 & 50.34 & JABEWN000000000 \\
\hline NJLSYY40 & 126 & 4964668 & 50.65 & JABEWL000000000 \\
\hline NJMJYY4 & 105 & 5024932 & 50.45 & JABEWH000000000 \\
\hline NJSDYYY42 & 139 & 5054242 & 50.76 & JABEWL000000000 \\
\hline NJSETYYY11 & 90 & 4884617 & 50.56 & JABEWM000000000 \\
\hline NJSETYYY14 & 112 & 4998406 & 50.60 & JABEWJ000000000 \\
\hline NJSFYBJY17 & 127 & 4768034 & 50.56 & JABEWK000000000 \\
\hline NJGLYY3610 & 194 & 5105893 & 50.73 & RZMI00000000 \\
\hline NJGLYY3940 & 210 & 5874804 & 50.85 & RZMM00000000 \\
\hline NJGLYY4197 & 3342 & 5786687 & 51.08 & JACKXC000000000 \\
\hline NJGLYY4673 & 66 & 5013086 & 50.69 & JACKXB000000000 \\
\hline
\end{tabular}

The Whole Genome Shotgun BioProject for these CREC isolates has been deposited at GenBank,

Table 3. The distribution of plasmid mediated quinolone genes, Virulence factors and plasmid replicons among the 11 carbapenem resistant Escherichia coli isolates from 6 hospitals in Nanjing. 


\begin{tabular}{|c|c|c|c|}
\hline Strains & PMQRs & Virulence factors & Plasmid replicons \\
\hline NJMJYY4 & $o q \times A B$ & ast $A$, gad & IncFIl, Incl1, IncX3, p0111 \\
\hline NJSETYYY11 & qnrS1 & IpfA & IncFIA, IncFIB, IncX3 \\
\hline NJSETYYY 14 & & IpfA & IncFII, Col(BS512), IncFIA, IncX3囚IncB/O/K/Z, IncFIB \\
\hline NJSFYYY17 & & gad & $\operatorname{IncX} 3$ \\
\hline NJLSYY40 & & gad $\ \mathrm{cma} \llbracket i s s \square / p f A \square a s t A$ & IncFIB, IncFIC(FII) $ه \operatorname{lncX} 3$ \\
\hline NJSDYYY42 & $q n r S 2, \operatorname{aac}\left(6^{\prime}\right) I b-c r$ & iroN $₫$ iss $\square \mathrm{cma} \square / p f A \square \mathrm{gad}$ & IncFII, IncFIB, IncFIC, IncX1, IncX3ХIncN \\
\hline NJJYYY51 & $q n r S 1$, oqxAB, $\operatorname{aac}\left(6^{\prime}\right) l b-c r$ & Iss, gad, IpfA & IncFII, IncFII(K), IncHI2, IncHI2A, IncX3 \\
\hline NJGLYY3610 & qnrS1 & iss, gad, capU & IncFII, IncFII, IncFIB, Incl1, IncX3, IncY \\
\hline NJGLYY 3940 & $q e p A, q n r A 1, \operatorname{aac}\left(6^{\prime}\right) l b-c r$ & gad, iroN, gad, cma, capU, iss & IncFII, IncFIB, IncY, IncX3 \\
\hline NJGLYY 4197 & qnrS1, aac $\left(6^{\prime}\right) I b-c r$ & sat, sen $B$, gad, iss, sepA, iha, cnf1, IpfA & IncFII, IncFIB, IncFII, ColpVC, IncHI1B, IncX1, IncX3, Col156 \\
\hline NJGLYY 4673 & $o q \times A B$ & ast $₫ \square g a d \rrbracket i s s \square / p f A \square c a p U$ & IncFII, IncFIA, IncFIB, IncHI2A, IncHI2, Incl1, IncX3 \\
\hline
\end{tabular}

PMQRs: plasmid mediated quinolone genes.

\section{Figures}

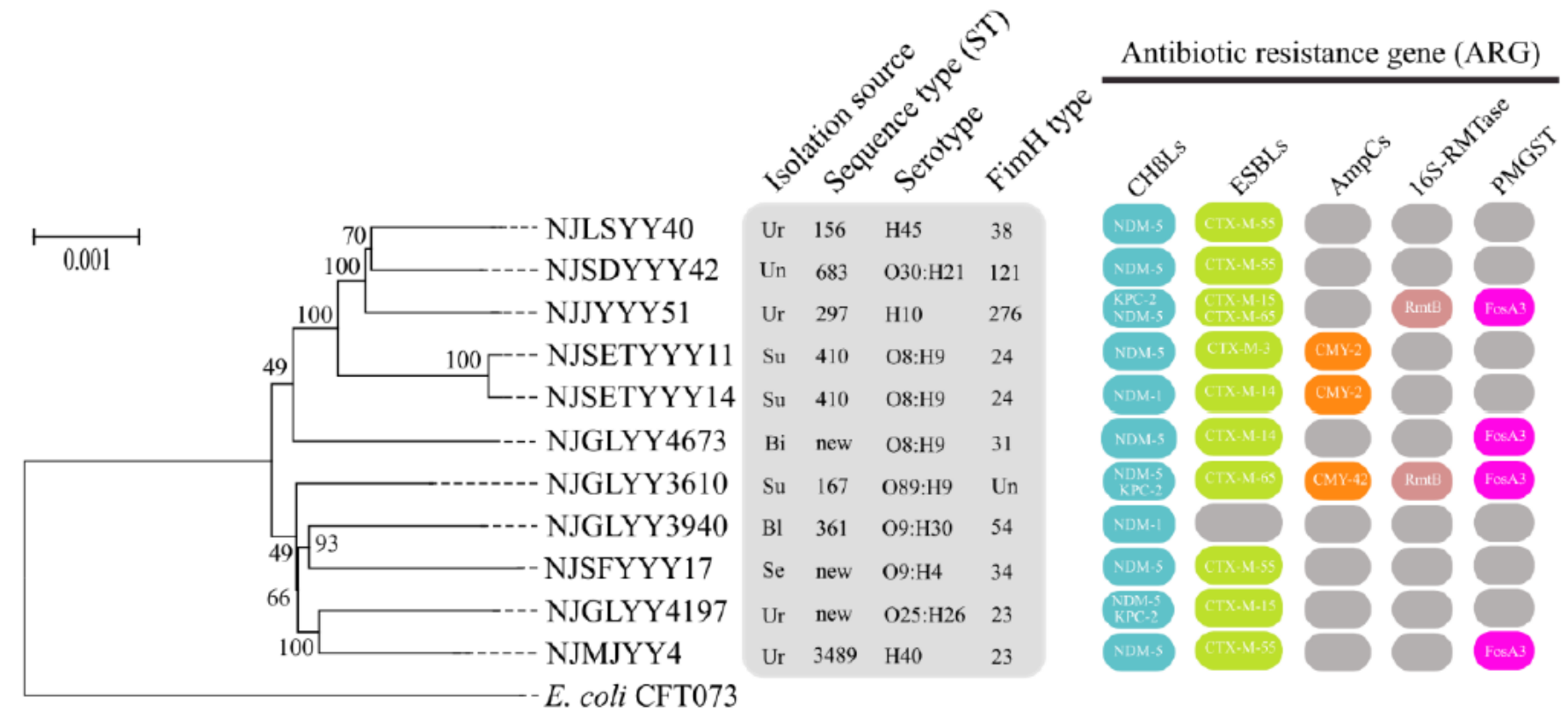

Figure 1

The heatmap of the 11 carbapenem resistant Escherichia coli isolates from 6 hospitals in Nanjing City. CH $\beta$ Ls: Carbapenem hydrolysis $\beta$-lactamase; ESBLs: extended-spectrum $\beta$-lactamase; pAmpCs: plasmid-mediated AmpC enzyme; 16S-RMTase: exogenously acquired 16S rRNA methyltransferase; PMGST: plasmid-mediated glutathione S-transferase. 


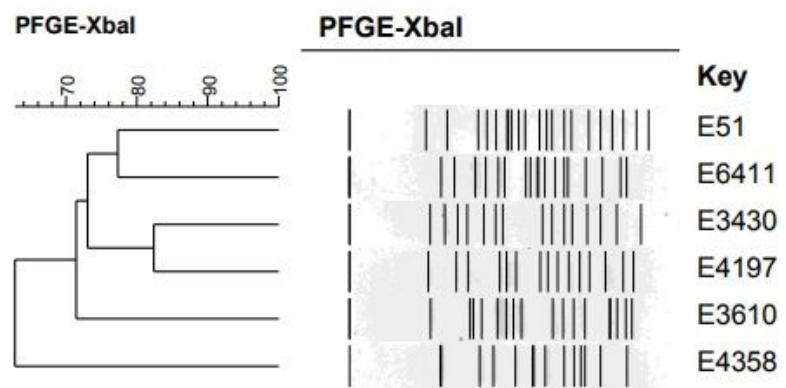

Origin
Urine
Sputum
Urine
Urine
Sputum
Abdominal fluid

$\begin{array}{ll}\text { CH } \beta \text { Ls } & \text { Year } \\ \text { NDM-5/KPC-2 } & 2015 \\ \text { NDM-5/KPC-2 } & 2017 \\ \text { NDM-5/KPC-2 } & 2014 \\ \text { NDM-5/KPC-2 } & 2017 \\ \text { NDM-5/KPC-2 } & 2015 \\ \text { NDM-1/KPC-2 } & 2015\end{array}$

Figure 2

Dendrogram based on PFGE profiles of 6 KPC-2 and NDM co-producing Escherichia coli isolates. The dendrogram was produced by the UPGMA algorithm based on the Dice similarity coefficient included five PFGE groups as defined based on $85 \%$ similarity of PFGE profiles. CHßLs, carbapenem hydrolyzing ßlactamase. 